\title{
UTILIZACIÓN DE SERIES TEMPORALES DE ÍNDICES ESPECTRALES BASADOS EN TELEDETECCIÓN PARA EL MONITOREO DE CULTIVOS DE TOMATE SOLANUM LYCOPERSICUM
}

\section{USE OF TIME SERIES OF SPECTRAL INDICES BASED ON REMOTE SENSING FOR MONITORING SOLANUM LYCOPERSICUM TOMATO CROPS}

\author{
Edier Fernando Ávila ${ }^{a, b,{ }^{*},}$, Bibiana Royero ${ }^{b}$ \\ ${ }^{a}$ Departamento de Ingeniería Topográfica y Cartográfica, Escuela Técnica Superior de Ingenieros en Topografía, Geodesia y \\ Cartografía, Universidad Politécnica de Madrid, Campus Sur, A-3, Km 7, 28031 Madrid, España. edierfernando.avila@alumnos.upm.es

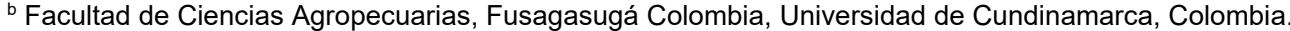 \\ efernandoavila@ucundinamarca.edu.co; broyero@ucundinamarca.edu.co
}

\begin{abstract}
:
The cultivation of tomato solanum lycopersicum is of great economic importance in Colombia, being Cundinamarca the fifth producing department of the country with an average of 371,827 tons for the year 2019 . New emerging technologies such as precision agriculture applied to the agricultural sector represent an opportunity to monitor physiological processes. Through the application of Remote Sensing with aerial images taken by UAV unmanned aerial vehicles and time series calculations of plant indices such as the Green Chlorophyll Index $(\mathrm{GCl})$, information on the production potential of the crop was quantified. The objective of this research was to extract relevant information from the production process in the batches, using Geomatic techniques. As results, the main batches with the highest production were identified, based on photosynthetic activity and growth height using a digital surface model, generated from the information obtained by photogrammetric flights. It was also possible to identify some microclimates generated in the lots, as a consequence of the difference in height found in the study area. As a final conclusion of achievement, obtain information and current characteristics of the plantation in order to make decisions in the next plantings.
\end{abstract}

Key words: remote sensing, plant indices, precision agriculture

\section{Resumen:}

El cultivo de tomate solanum lycopersicum es de gran importancia económica en Colombia, siendo Cundinamarca el quinto departamento productor del país con un promedio de 371.827 toneladas para el año 2019. Las nuevas tecnologías emergentes como la agricultura de precisión aplicada al sector agrícola, representan una oportunidad para monitorear procesos fisiológicos. Mediante la aplicación de la teledetección con imágenes aéreas tomadas por vehículos aéreos no tripulados UAV y cálculos de series de tiempo de índices vegetales como el Green Chlorophyll Index (GCI), se cuantificó información sobre el potencial de producción del cultivo. El objetivo de la presente investigación fue extraer información relevante del proceso productivo en los lotes, mediante tecnicas geomáticas. Como resultados se identificaron los principales lotes con mayor producción, basados en la actividad fotosintética y la altura de crecimiento utilizando un modelo digital de superficie, generado partir de la información obtenida por los vuelos fotogramétricos. También se pudo identificar algunos microclimas generados en los lotes, como consecuencia de la diferencia de altura encontrada en la zona de estudio. Como conclusión final se logró obtener información y características actuales de la plantación con el fin de tomar decisiones en las próximas siembras.

Palabras clave: teledetección, índices vegetales, agricultura de precisión

\section{Introducción}

La gestión de la información geográfica ha permeado casi todas las áreas de las ciencias tanto básicas como aplicadas. La consecución de los datos geo científicos en el campo de la agricultura, está tomando gran relevancia para la toma de decisiones a nivel de producción y optimización del recurso, tanto ambiental físico y económico.

Según pronosticó la $\mathrm{FAO}$, será necesario aumentar la producción de alimentos para el 2050 en un $70 \%$ y en los países en vías de desarrollo casi duplicarse para responder al crecimiento poblacional estimado en 9100 millones de personas (Radoglou-Grammatikis et al. 2020).

Para ello, los agricultores, las agremiaciones, la comunidad de investigación agrícola, y las empresas en general, requieren acceso a herramientas de alta precisión. Especialmente analizar y estimar áreas productivas, facilitar la toma de decisiones tempranas en el desarrollo de los cultivos (fertilización, podas, manejo sanitario) y obtener con ello, un mayor rendimiento

`Corresponding Author: Edier Fernando Ávila, efernandoavila@ucundinamarca.edu.co 
productivo y sostenibilidad ambiental y social (Srivastava et al. 2019).

Hoy en día el seguimiento a los campos agrícolas en Colombia, y en otros países en vía de desarrollo se realiza de una manera artesanal y empírica, recurriendo a la experiencia de los agricultores y a su percepción visual de las deficiencias en el cultivo. La detección de alteraciones en los cultivos como plagas y enfermedades, requieren gran esfuerzo en tiempo y en recurso. Además, los profesionales capacitados en estas áreas son escasos y pueden tomar decisiones subjetivas en términos agronómicos. Los vehículos aéreos no tripulados (UAV), equipados con sensores multiespectrales, pueden disminuir el tiempo de recopilación de datos y reducir los costos operativos (Ampatzidis et al. 2020).

En la investigación realizada por (Deng et al. 2018), utilizaron cámaras multiespectrales para recopilar información del cultivo de maíz, se comparó las precisiones de varios índices vegetales, sin embargo existen limitaciones a nivel general de estas técnicas en la adquisición, el procesamiento y la generación de datos.

Se reportan estudios con diseños experimentales en el campo de la agricultura de precisión, donde utilizando UAV, se obtuvo información a nivel submétrico del orden de 10 centímetros de píxel (Rokhmana 2015). Esta resolución espacial es ideal para el seguimiento y monitoreo de la salud de las plantas (Rokhmana 2015). También se reportan resultados a partir de modelos digitales de elevación (DEM), por sus siglas en inglés (Doering et al. 2014), para evaluar la topografía y la configuración del terreno donde se ubican plantaciones agrícolas.

Investigaciones recientes arrojan resultados donde identifican la calidad de las hojas en un cultivo, detectando hongos, y áreas infectadas mediante levantamientos topográficos, basado en el reconocimiento de patrones espectrales. Principalmente mediante la recolección de fotografías, que son tomadas por UAVs (Nakshmi et al. 2020). Los resultados arrojados detectan malezas, hojas secas y sanas en función de su actividad fotosintética.

Se puede estimar el vigor vegetativo de los cultivos a partir de índices vegetales I. V. y capas topográficas, lo cual permite resaltar aquellas áreas donde las plantas gozan de una alta actividad fotosintética (Modica et al. 2020). Se ha establecido diferentes I. V. para cultivos determinados, entre ellos está el índice, Green Chlorophyll Index $(\mathrm{GCl})$. Esta técnica demuestra el potencial para rastrear remotamente el estado fisiológico de los cultivos (Gitelson et al. 2005).

En Argentina, mediante el sistema de telemetría, analizaron el manejo del riego por goteo de una parcela experimental del cultivo de tomate, en el que se aplicaron imágenes termográficas y multiespectrales para apreciar condiciones de estrés hídrico en el cultivo aplicando Índices de vegetación diferenciada normalizada (NDVI), (Caparro et al. 2019).

En Colombia, las investigaciones y aplicaciones en el área agricultura de precisión son muy escasas, solo algunos centros investigativos y universidades han desarrollado algunas técnicas de monitoreo (Ávila et al. 2019).
La presente investigación pretende analizar mediante una serie de tiempo de imágenes tomadas por UAV, el índice de vegetación $\mathrm{GCl}$ aplicado a una plantación de tomate (Solanum lycopersicum). Este índice aporta información relevante en el comportamiento de la actividad fotosintética de los cultivos, lo cual repercute en la producción agrícola de la parcela (Gitelson et al. 2005).

\section{Método}

La zona de estudio se ubica en el municipio de Fusagasugá y Silvania Cundinamarca, con coordenadas geográficas latitud 4.344 grados decimales y longitud oeste de -74.385 grados decimales. Posee una topografía relativamente plana y un área aproximada de 2.47 Hectáreas dividida en 12 lotes productivos.

Se utilizó un cuadricoptero agrícola de la serie Parrot Bluegrass Fields, con cámara multiespectral Parrot Sequoia. Este instrumento contiene cuatro bandas multiespectrales en verde $550 \mathrm{~nm}$, rojo $660 \mathrm{~nm}$, borde rojo $735 \mathrm{~nm}$ e infrarrojo cercano con $790 \mathrm{~nm}$.

\subsection{Georeferenciación de la parcela}

Como primera etapa de la investigación, se garantizó la exactitud posicional y altimétrica (altura elipsoidal) de los diferentes vuelos realizados. Es así, que se navegaron y materializaron cuatro puntos con tecnología de sistema de navegación global por satelital GNSS, los cuales se ajustaron con archivos Rinex (Receiver INdependent EXchange) de la estación Boga (IGAC 2021) perteneciente al Instituto geográfico Agustín Codazzi IGAC (Fig. 1).

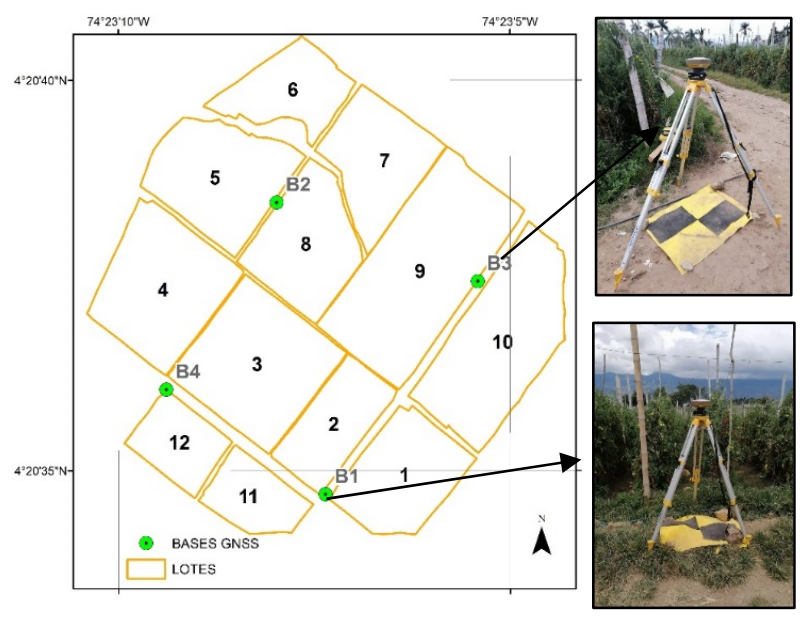

Figura 1: Descripción general de la zona de estudio y ubicación de los puntos GNSS.

Para la adquisición de los datos GNSS se utilizaron antenas Topcon de la serie Hiper SR, las cuales son de una frecuencia $L 1$, y pueden recolectar datos de las constelaciones GPS y GLONAS.

\subsection{Postproceso de los datos navegados GNSS}

El postproceso de los datos navegados con las antenas Hiper SR (Topcon 2021), se realizó en el software Topcon Tools. Cada punto se navegó aproximadamente tres horas que es el estándar según la normatividad del IGAC para Colombia. En la Figura 2 se observa el tiempo de 
recepción de la señal en las Bases $B 1$ y $B 2$. A postprocesar los cuatro puntos B1, B2, B3, B4, se obtuvieron muy buenos resultados en planimetría y la altura elipsoidal, según se muestra en la Tabla 1.

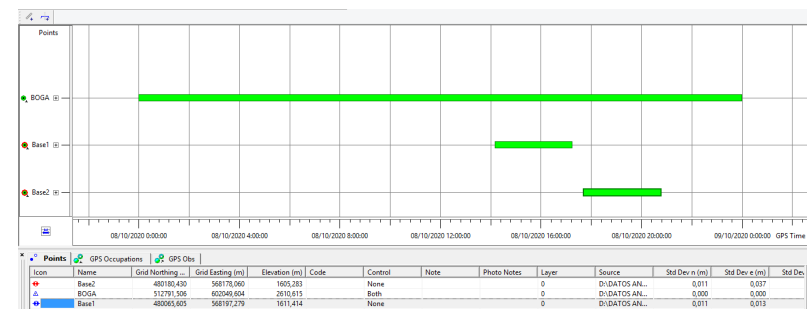

Figura 2: Visual del ajuste a la navegación de los puntos B1 y B2 en software Topcon Tools.

Tabla 1: Resultados de las coordenadas de los cuatro puntos navegados ya ajustados.

\begin{tabular}{c|c|c|c|c|c} 
Base & Norte $(m)$ & Este $(m)$ & $\begin{array}{c}\text { Elevación } \\
(m)\end{array}$ & $\begin{array}{c}\text { Horz } \\
\text { RMS } \\
(m)\end{array}$ & $\begin{array}{c}\text { Vert } \\
\text { RMS } \\
(m)\end{array}$ \\
\hline B1 & 480065.60 & 568197.27 & 1611,41 & 0.01 & 0.02 \\
B2 & 480180.43 & 568178.06 & 1605.28 & 0.03 & 0.02 \\
B3 & 480149.44 & 568257.18 & 1612.56 & 0.01 & 0.02 \\
B4 & 480106.80 & 568134.64 & 1609.98 & 0.02 & 0.03
\end{tabular}

Los datos de la Tabla 1, son importantes dentro de la generación de los ortofotomosaicos de cada vuelo fotogramétrico, con el fin de buscar una relación espacial de superposición temática pixel a pixel. Se observa en los datos de la Tabla 1, que poseen una exactitud milimétrica con un máximo de error en el punto B3 de 3 centímetros en planimetría.

\subsection{Realización de los vuelos fotogramétricos}

Una vez obtenidas las coordenadas ajustadas, se procede con la realización de los vuelos fotogramétricos sobre el cultivo de tomate. En la Tabla 2, se observa las fechas de realización, partiendo como fecha de siembra del cultivo la segunda semana del mes de mayo del 2020.

Para la realización de los vuelos fotogramétricos, se garantizó en lo posible tener las mismas condiciones atmosféricas y técnicas para el pilotaje de la aeronave, la cual se realizó de forma automática, teniendo los mismos parámetros de recubrimiento o traslape tanto horizontal como vertical. También se conservó la misma resolución espacial y líneas de vuelo como se observa en la Figura 3 y Tabla 2.

\subsection{Cálculo del índice vegetal GCI}

Después de realizado cada vuelo fotogramétrico, se calculó el índice vegetal Green Chlorophyll Index (GCl) (Ec. 1). Este índice según varios estudios, es un buen indicador del contenido de clorofila en el dosel (Wu et al. 2012). El índice relaciona las bandas verdes e infrarrojas cercanas, lo cual, es importante en el monitoreo de la actividad fotosintética de las plantas (Gitelson et al. 2005). Los mapas derivados de este índice pueden observarse en la Figura 4.

$G C I=\left(\frac{N I R}{V E R D E}\right)-1$
Donde:

$N I R=$ Longitud de onda del Infrarrojo cercano $V E R D E=$ Longitud de onda del verde

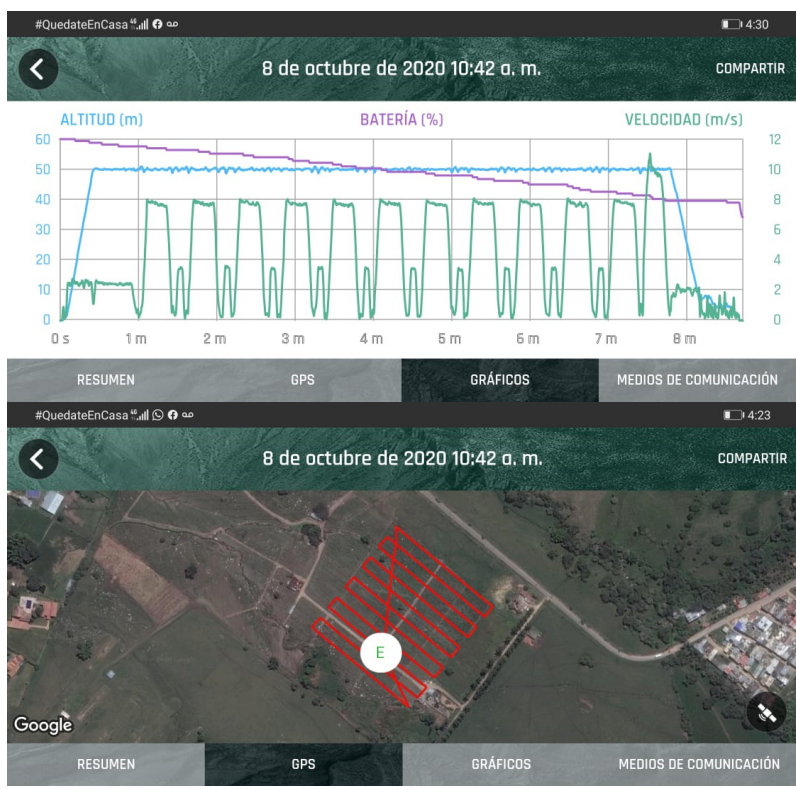

Figura 3: Visual del plan de vuelo y parámetros de las líneas de vuelo sobre el cultivo de tomate.

Tabla 2: Descripción de los seis vuelos realizados en el cultivo de tomate.

\begin{tabular}{c|c|c|c|c|c} 
Vuelo & Fecha & Fotografías & Altura $(m)$ & $G S D(m)$ & Días \\
\hline 1 & $23 / 06 / 2020$ & 600 & 50 & 0.06 & 0 \\
2 & $07 / 07 / 2020$ & 912 & 40 & 0.04 & 14 \\
3 & $31 / 07 / 2020$ & 524 & 60 & 0.06 & 24 \\
4 & $24 / 09 / 2020$ & 796 & 60 & 0.06 & 55 \\
5 & $08 / 10 / 2020$ & 752 & 50 & 0.05 & 14 \\
6 & $13 / 10 / 2020$ & 1104 & 50 & 0.05 & 5
\end{tabular}

En la anterior serie temporal del índice vegetal $\mathrm{GCI}$ (Ec. 1), se observa el comportamiento espectral vegetativo del cultivo de tomate. A mayor valor se deduce que contiene una alta actividad fotosintética, lo que se observa en el vuelo 2 (Fig. 4b), donde hay un máximo visual de contraste del contenido de clorofila.

\subsection{Generación del modelo digital de superficie (DSM)}

Con los puntos georeferenciados B1, B2, B3, B4 y una exactitud milimétrica, se generó en la investigacion el Modelo Digital de Superficie MDS de la zona de cultivos. Esta información es de vital importancia ya que se puede correlacionar la actividad fotosintética con la altura que desarrolla la planta, consiguiendo realizar predicciones de rendimiento (Maes and Steppe 2019). 


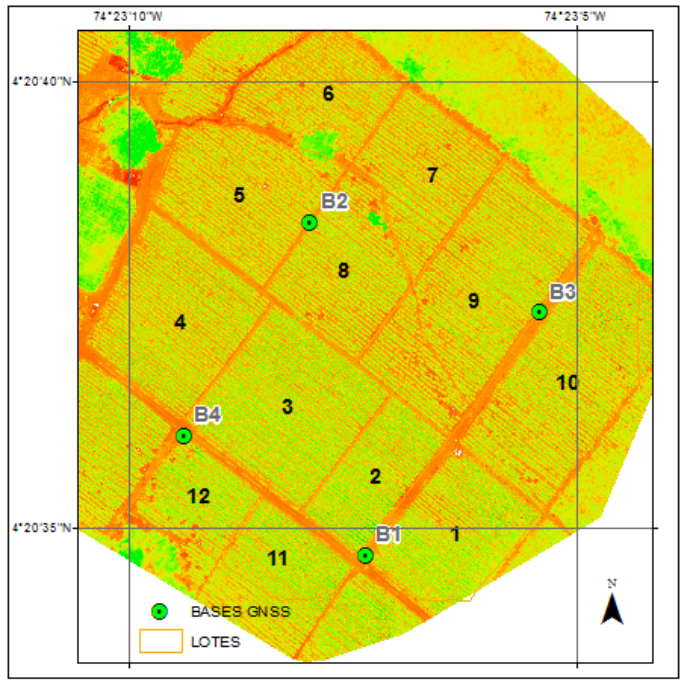

(a)

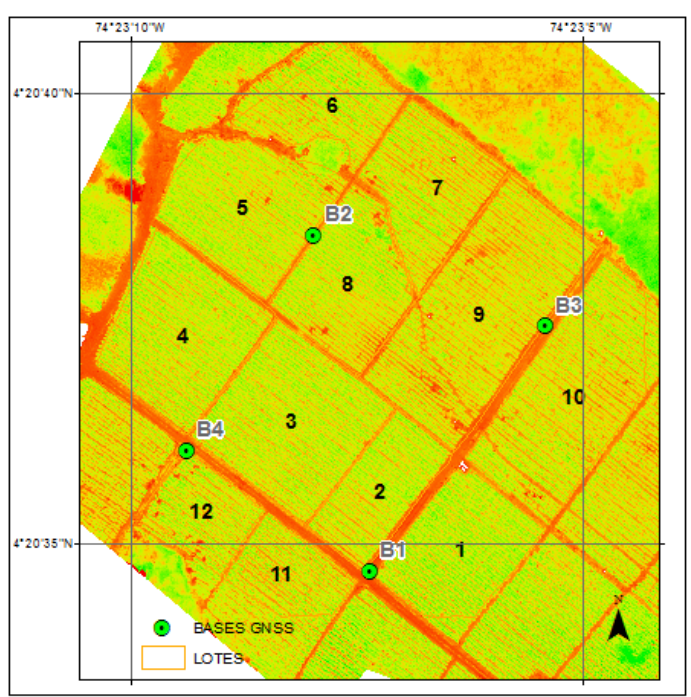

(c)

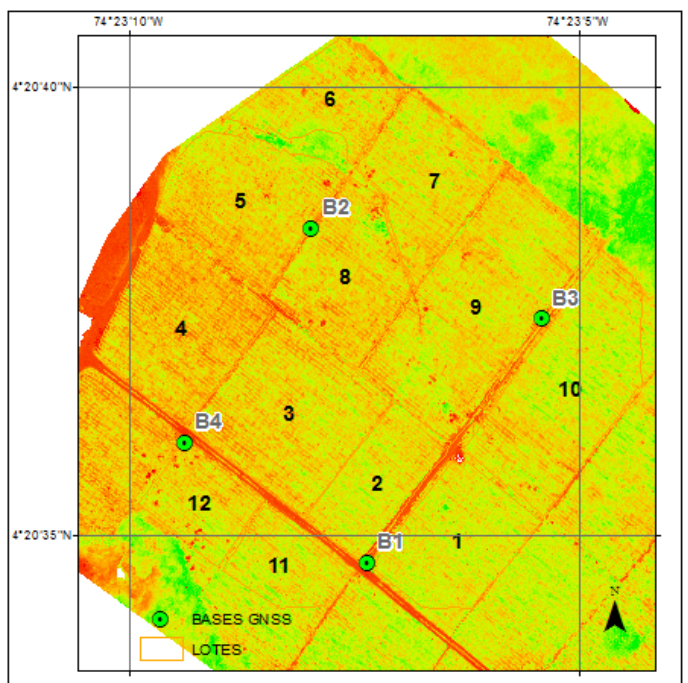

(e)

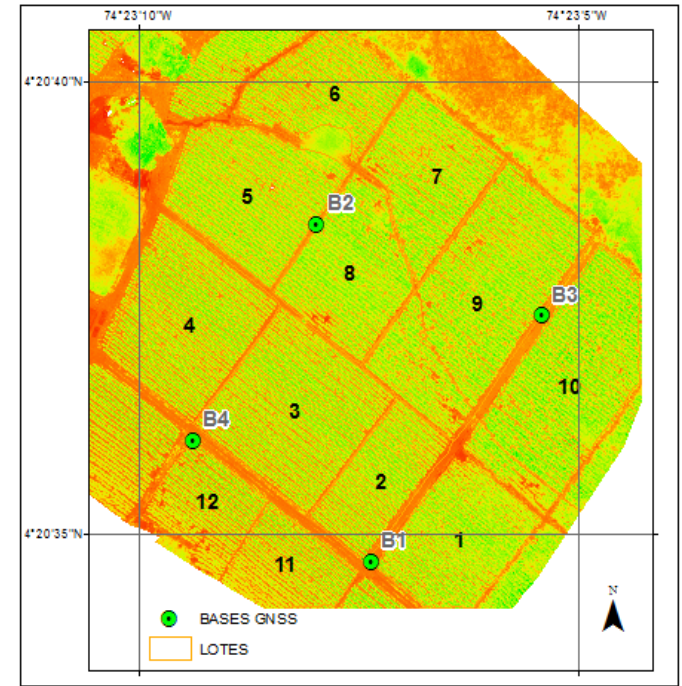

(b)

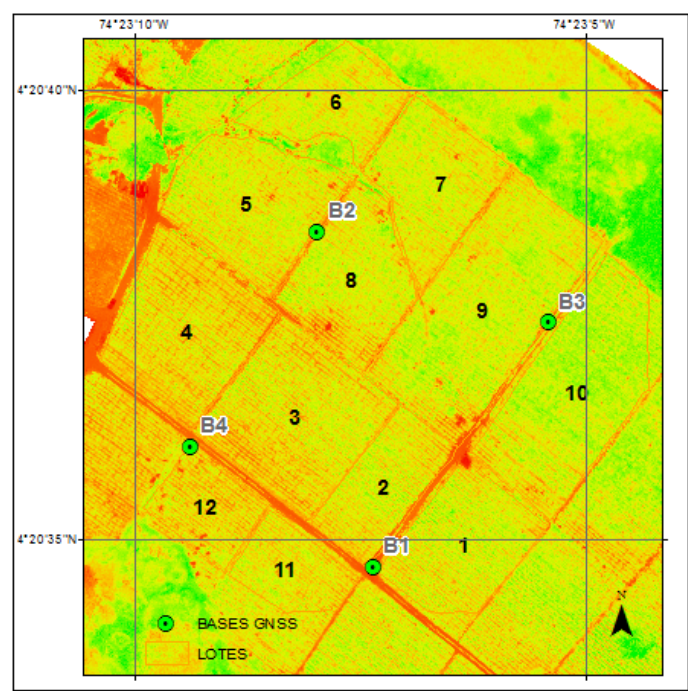

(d)

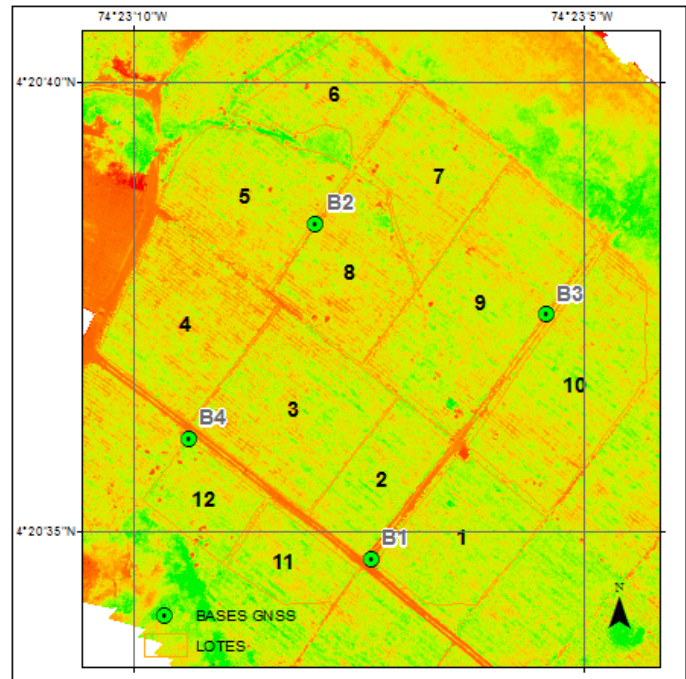

(f)

Figura 4: Visual del cálculo de la serie de tiempo del índice Vegetal GCl: a) Vuelo 1; b) Vuelo 2; c) Vuelo 3; d) Vuelo 4; ) Vuelo 5; f) Vuelo 6. 


\section{Resultados y discusion}

Como resultado principal de la investigación se obtiene el promedio del comportamiento o caracterización de índice vegetal $\mathrm{GCl}$ (Ec. 1), en los diferentes momentos o series de tiempo por cada lote productivo. Una alta actividad fotosintética, información que arroja el estudio puede asociarse a una mayor productividad. Esta información es relevante en cuanto a las variaciones del contenido de clorofila que podrían predecir el rendimiento de los lotes de cultivo.

El valor del índice $\mathrm{GCl}$ es variable durante el periodo del cultivo en función al estado fenológico de las plantas y las diferentes variables ambientales, nutricionales, sanitarias que afectan al mismo, entre otras.

Se evidencia, en las Figuras 4 y 5 una correlación visual entre la actividad clorofílica de los lotes de mayor productividad y la altura de la planta. Para esta investigacion no se tomaron datos de luminosidad o radiación solar, sería interesante para próximas aplicaciones tener en cuenta esta variable. Se infiere que los lotes con un desnivel topográfico más bajo o menos altura elipsoidal, tienden a tener menor valor de $\mathrm{GCl}$, por ejemplo el lote 4 tuvo una suma total de $\mathrm{GCl}$ de 16 , con respecto al lote 2 que tuvo un valor de 18.1, según la Tabla 3. Estos valores son adimensionales, lo que presume la generación de microclimas dentro de todo el sembradío. Estos desniveles pueden conllevar una alta humedad que favorece procesos infectivos y ciclos reproductivos de hongos como Mildiu Phytophthora infestans y bacterias como la peca bacteriana Pseudomonas syringae presentes en este cultivo. Esto afecto severamente la producción de algunos lotes como se evidencia en la Tabla 3, con los lotes 9, 12 y 4.

Tabla 3: Ubicación del lote en función del valor del Índice vegetal GCl.

\begin{tabular}{c|c|c|c|c|c|c|c|c} 
Lote & $V 1$ & $V 2$ & $V$ 3 & $V 4$ & $V$ 5 & $V$ 6 & Suma GCI & Prod. (\%) \\
\hline L1 & 2.4 & 3.7 & 3.9 & 3.2 & 3.1 & 3.0 & 19.6 & 100.0 \\
L10 & 2.1 & 3.9 & 3.3 & 3.6 & 3.0 & 2.7 & 18.8 & 96.0 \\
L2 & 2.4 & 3.2 & 3.5 & 3.0 & 2.9 & 2.8 & 18.1 & 92.7 \\
L11 & 2.6 & 2.9 & 3.3 & 2.9 & 3.0 & 2.9 & 17.9 & 91.4 \\
L5 & 1.9 & 3.6 & 3.6 & 3.1 & 2.6 & 2.5 & 17.5 & 89.7 \\
L6 & 1.8 & 3.3 & 3.4 & 3.2 & 2.9 & 2.7 & 17.5 & 89.6 \\
L3 & 2.4 & 3.3 & 3.6 & 2.7 & 2.6 & 2.6 & 17.4 & 89.1 \\
L7 & 1.8 & 3.5 & 3.2 & 3.1 & 2.8 & 2.5 & 17.2 & 88.0 \\
L8 & 2.0 & 3.7 & 3.4 & 3.0 & 2.6 & 2.3 & 17.2 & 87.8 \\
L9 & 1.9 & 3.5 & 2.9 & 3.2 & 2.7 & 2.6 & 17.1 & 87.6 \\
L12 & 2.4 & 2.8 & 3.3 & 2.6 & 2.8 & 2.8 & 17.0 & 86.7 \\
L4 & 2.0 & 3.1 & 3.6 & 2.6 & 2.2 & 2.3 & 16.0 & 82.0
\end{tabular}

Como resultado de la investigación, los lotes más productivos tuvieron mayores valores del índice vegetal $\mathrm{GCl}$ o fueron estables en un periodo de tiempo. Aplicando la Ec. (2), se obtiene la Tabla 3.

$\lim _{G C I \rightarrow \infty} \sum_{n=1}^{6} \mathrm{GCIVn} \approx$ Alta productividad del lote
Donde:

$G C I V n=\mathrm{El}$ valor del índice $\mathrm{GCl}$ en el vuelo $n$ por cada lote de producción

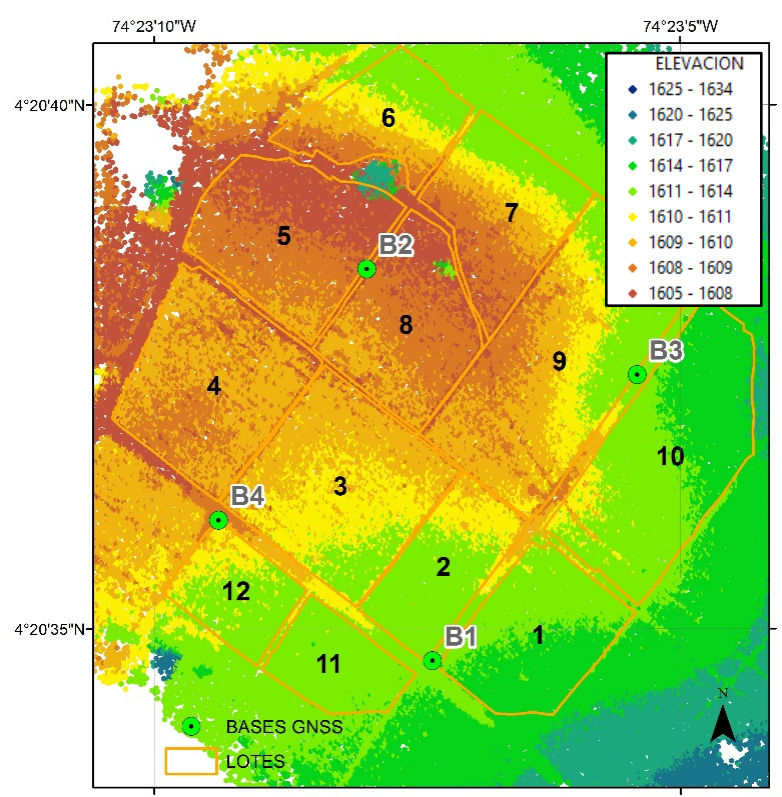

Figura 5: Modelo Digital de Elevación zona de estudio.

En el desarrollo de la formula anterior, se sumaron los valores promedios del índice $\mathrm{GCl}$, en cada lote por cada vuelo, y la connotación de "limite", se aplica porque no conocemos un valor máximo del índice y lo que se busca, es encontrar ese máximo que traduce en una alta actividad fotosintética.

Como resultado de (2), se caracterizó los lotes de mayor porcentaje de producción en función del índice $\mathrm{GCl}$, como lo muestra la Tabla 3 y Figura 5.

La columna Prod. de la Tabla 3, está calculada porcentualmente, en función del lote de máximo valor del índice $\mathrm{GCl}$, asociando el valor de mayor índice como el $100 \%$.

Este resultado fue contrastado con el administrador del cultivo, quien validó la información confirmando a los lotes 1 y 10 como "los más productivos". No fue posible obtener los valores de la producción por lote, ya que el mecanismo de cosecha del tomate es el número de kilos/finca y no la producción de cada lote de cultivo.

Se graficaron los promedios de $\mathrm{GCl}$ por cada lote, obteniendo una serie temporal, que puede observarse en la Figura 6. En esta gráfica se evidencia una tendencia a la baja del valor del índice $\mathrm{GCl}$ en particular para los últimos vuelos que coincide con el ciclo del cultivo. Esta informacion se genera como resultado del cambio en la edad fisiológica de la planta, la cual a medida que envejece disminuye su capacidad para realizar fotosíntesis y acelera la degradación de las clorofilas (Barraza et al. 2004). Las hojas jóvenes se convierten en depósito preferencial para donde se exportan los nutrimentos, limitándolos en las hojas más senescentes (Cayón 1992; Morales 2013). 


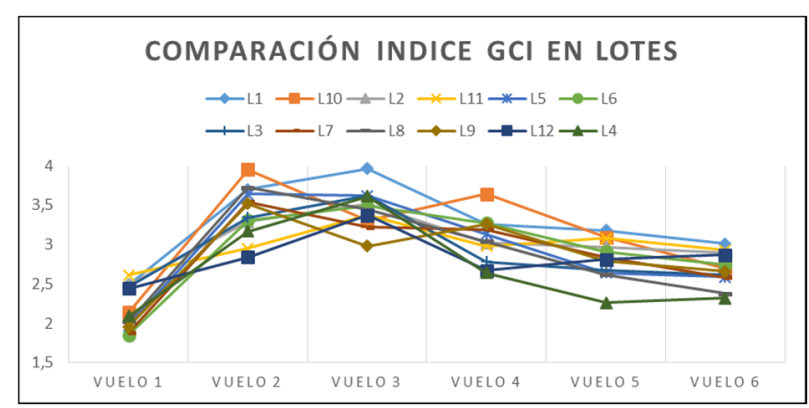

Figura 6: Serie temporal del índice $\mathrm{GCl}$ en los diferentes vuelos fotogramétricos.

Como se observa en la Figura 6, en el vuelo 2 de fecha 07/07/2020, el cultivo de tomate tiene 70 días de sembrado aproximadamente. En esta etapa del crecimiento de su fase vegetativa y de desarrollo floral alcanza su máxima actividad fotosintética según los datos del GCl. Después de este periodo comienza un descenso paulatino, que coincide con la aparición de problemas fitosanitarios en el cultivo y posteriormente con etapas fenológicas de menor eficiencia fotosintética.

En la Figura 7, se grafica una primera aproximación a la serie temporal del Índice Vegetal $\mathrm{GCl}$, del cultivo de tomate. Estos datos modelan el comportamiento del índice $\mathrm{GCl}$ en función del crecimiento del cultivo. Este resultado es relevante para próximas cosechas, ya que se puede inferir una producción aproximada, según el valor del índice $\mathrm{GCl}$. Se recomienda para próximos estudios de índices, tomar varios datos temporales, con el fin de obtener una curva robusta estadísticamente.

\section{SERIE TEMPORAL GCI}

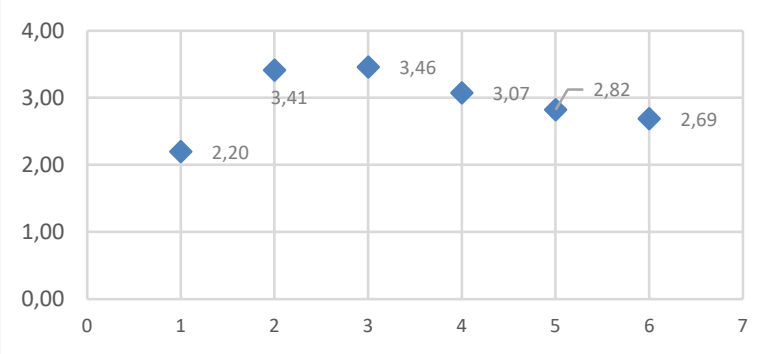

Figura 7: Aproximación de la función Índice Vegetal GCI.

Es importante destacar que esta investigación se realizó en un monocultivo de tomate a libre exposición destinado a la producción a pequeña escala y no a la investigación. Debido a esto, no se controlaron algunas variables como la prescripción agronómica, dirección de los surcos en relación a factores medioambientales, el manejo nutricional y fitosanitario, la aplicación de fitohormonas entre otros. Estas variables dificultan la interpretación de los cambios en los resultados de la información espectral, y por ende limitan en alguna medida los alcances de esta herramienta en la gestión del cultivo. Por ello, es recomendable incorporar el uso de sensores climaplanta-suelo instalados directamente en campo, para obtener mayor información, que facilite la toma de decisiones de los agricultores y agrónomos en campo.

\section{Conclusiones}

Con el desarrollo de la presente investigacion, se realiza un aporte a las técnicas de la agricultura de precisión, Este aporte consisten en modelar una producción agrícola en función de la intensidad de la energía radiada por el cultivo. Con esta información los agricultores pueden monitorear la actividad fotosintética, y tomar decisiones a tiempo en cuanto a su fertilización y aplicación de agroquímicos, con el fin obtener una producción máxima en los cultivos de tomate solanum lycopersicum.

Se propone tomar imágenes con mayor frecuencia, antes de los 60 días después de la siembra, ya que los datos muestran una tendencia rápida al incremento de la actividad fotosintética, y es en esta parte del proceso productivo cuando se debe tener precaución en el cuidado y fortalecimiento de la planta.

Con la realización de este tipo de investigación, que son novedosas, en el campo de la agricultura en Colombia y más exactamente en Silvania, se realiza un aporte a la planificación y racionamiento en la aplicación de agroquímicos para el control de plagas y enfermedades en los cultivos, disminuyendo los contaminantes generados por la actividad agrícola.

\section{Agradecimientos}

Se agradece al grupo de investigación geocartografia, y al proyecto de investigacion "Herramienta geoespacial para la construcción del diagnóstico socioambiental del Plan de Desarrollo Territorial del municipio de Silvania Cundinamarca.", código 63546, convocatoria Colciencias 2018.

\section{References}

AMPATZIDIS, Y., PARTEL, V., and COSTA, L., 2020. Agroview: Cloud-based application to process, analyze and visualize UAV-collected data for precision agriculture applications utilizing artificial intelligence. Computers and Electronics in Agriculture, 174(February), 105457. DOI: 10.1016/j.compag.2020.105457

ÁVILA, E. F., ESCOBAR, N., and MORANTES, C. F., 2019. Applying satellite images to spectral signature development of maize production (Zea mays L.) under colombia's middle tropics conditions. Entramado, 15(2), pp. 256-262. DOI: 10.18041/1900-3803/entramado.2.5734

BARRAZA, F. V., FISCHER, G., and CARDONA, C. E., 2004. Estudio del proceso de crecimiento del cultivo del tomate (Lycopersicon esculentum Mill.) en el Valle del Sinú medio, Colombia. Agronomía Colombiana, 22(1), pp. 81-90.

CAPRARO, F., TOSETTI, S., MUT, V., CAMPILLO, P., OLGUÍN, A., and PACHECO, D., 2019. Implementación del sistema "Telemetría Agrícola" para el manejo y análisis de estrategias de riego deficitario controlado en el cultivo de tomate. In XI Congreso de Agrolnformática (CAI)-JAIIO 48 (Salta, 2019). 
CAYÓN, G., 1992. Fotosíntesis y productividad de cultivos. Revista COMALFI 19(2), pp. 23-31.

DENG, L., MAO, Z., LI, X., HU, Z., DUAN, F., and YAN, Y., 2018. UAV-based multispectral remote sensing for precision agriculture: A comparison between different cameras. ISPRS Journal of Photogrammetry and Remote Sensing, 146(September), pp. 124-136. DOI: 10.1016/j.isprsjprs.2018.09.008

DOERING, D., BENENMANN, A., LERM, R., DE FREITAS, E. P., MULLER, I., WINTER, J. M., and PEREIRA, C. E., 2014. Design and optimization of a heterogeneous platform for multiple UAV use in precision agriculture applications. IFAC Proceedings Volumes (IFAC-PapersOnline) (Vol. 19). DOI: 10.3182/20140824-6-za-1003.02261

GITELSON, A. A., VINA, A., CIGANDA, V., RUNDQUIST, D. C., and ARKEBAUER, T. J. (2005). Remote estimation of canopy chlorophyll content in crops. Geophysical Research Letters, 32(8). DOI: 10.1029/2005GL022688

IGAC 2021. Datos Abiertos IGAC | GEOPORTAL. Available: https://geoportal.igac.gov.co/contenido/geodesia-archivosen-formato-rinex-estaciones-red-magna-eco [1/09, 2021].

MAES, W. H., and STEPPE, K., 2019. Perspectives for Remote Sensing with Unmanned Aerial Vehicles in Precision Agriculture. Trends in Plant Science, 24(2), pp. 152-164. DOI: 10.1016/j.tplants.2018.11.007

MODICA, G., MESSINA, G., DE LUCA, G., FIOZZO, V., and PRATICÒ, S., 2020. Monitoring the vegetation vigor in heterogeneous citrus and olive orchards. A multiscale object-based approach to extract trees' crowns from UAV multispectral imagery. Computers and Electronics in Agriculture, 175(February), $105500 . \quad$ DOI: 10.1016/j.compag.2020.105500

MORALES, D., 2013. Efecto de altas temperaturas en algunas variables del crecimiento y el intercambio gaseoso en plantas de tomate (Lycopersicon esculentum Mill. cv. Amalia). Cultivos tropicales, 27(1), pp. 45-48.

NAKSHMI, J. V. N., HEMANTH, K. S., and BHARATH, J., 2020. Optimizing Quality and Outputs by Improving Variable Rate Prescriptions in Agriculture using UAVs. Procedia Computer Science, 167(Iccids 2019), pp. 1981-1990. DOI: 10.1016/j.procs.2020.03.229

RADOGLOU-GRAMMATIKIS, P., SARIGIANNIDIS, P., LAGKAS, T., and MOSCHOLIOS, I., 2020. A compilation of UAV applications for precision agriculture. Computer Networks, 172(February), $107148 . \quad$ DOI: 10.1016/j.comnet.2020.107148

ROKHMANA, C. A., 2015. The Potential of UAV-based Remote Sensing for Supporting Precision Agriculture in Indonesia. Procedia Environmental Sciences, 24, pp. 245-253. DOI: 10.1016/j.proenv.2015.03.032

SRIVASTAVA, K., BHUTORIA, A. J., SHARMA, J. K., SINHA, A., and PANDEY, P. C., 2019. UAVs technology for the development of GUI based application for precision agriculture and environmental research. Remote Sensing Applications: Society and Environment, 16(May), 100258. DOI: 10.1016/j.rsase.2019.100258

TOPCON 2021. HiPer SR - Receptor GNSS ligero y compacto | Topcon Positioning Systems, Inc. Available: https://www.topconpositioning.com/es/gnss-and-network-solutions/integrated-gnss-receivers/hiper-sr\#panel-productspecifications [1/09, 2021].

WU, C., NIU, Z., and GAO, S., 2012. The potential of the satellite derived green chlorophyll index for estimating midday light use efficiency in maize, coniferous forest and grassland. Ecological Indicators, 14(1), pp. 66-73. DOI: 10.1016/j.ecolind.2011.08.018 